\begin{tabular}{|c|c|c|c|c|c|}
\hline MUNIBE Antropologia-Arkeologia & $n^{\circ} 65$ & $37-51$ & DONOSTIA & 2014 & ISSN 1132-2217 • eISSN 2172-4555 \\
\hline
\end{tabular}

\title{
Mirando atrás: Las representaciones de zoomorfos retrospicientes en el arte paleolítico europeo
}

\author{
Looking back: The retrospiscens zoomorphic \\ representations in European Palaeolithic Art
}

KEY WORDS: Portable art, Rock Art, Upper Palaeolithic, bestiary, animation.

PALABRAS CLAVES: Arte mueble, arte parietal, Paleolítico superior, bestiario, animación

GAKO-HITZAK: Arte higigarria, arte parietala, Goi Paleolitikoa, bestiarioa, animazioa.

\section{Alberto LOMBO MONTAÑÉS(1), Clara HERNANDO ÁLVAREZ ${ }^{(2)}$ y Manuel BEA ${ }^{(3)}$}

\begin{abstract}
This paper provides a study of zoomorphic graphics with head turned back in the Palaeolithic art. The analysis of these figures allows a series of observations about their technology (orientation), chronology and contextual elements (thematic partnerships), while it approaches us their meaning. The zoomorphic figures with head turned back takes part of a small special group within the graphic repertoire of Palaeolithic art. It's a graphic motif that reflects true attitudes of the animals as well as they might have been a key element in the graphic transmission because of its large geographic dispersion.

In European Palaeolithic art, a small number of zoomorphic representations were represented with their head turned backwards. These zoomorphs (bison licking their back, red deer hinds with ears pricked up, caprids giving birth and defecating) have attracted the attention of researchers as they appear to be faithful reflections of certain animal behaviour, at the same time as they form a graphic model with a wide geographical distribution. However, this group of figures has never been studied as a whole, enabling an examination of this way of representing zoomorphs in its thematic, technical and technological aspects. This paper studies 55 "backward-looking" graphic units from portable and parietal ensembles attributed to the European Upper Palaeolithic. It covers their zoomorphic identification, the type of surface they are depicted on, the technique used and their chronological and geographical distribution. The results highlight the relative spatial diffusion of these motifs during the pre-Magdalenian period, when they are predominantly parietal horse and cervid representations, and the concentration of backward-looking depictions in Cantabrian Spain and the French Pyrenees during the middle Magdalenian technocomplex, the period to which nearly half the known examples are attributed.

Secondly, a new explanation is proposed for the production of some backward-looking representations whose head is depicted over their body. It has traditionally been accepted that this position was a consequence of the lack of space available to the artist. However, in some cases of enough space, the animals' heads were deliberately depicted inside the silhouette of the body, perhaps with the intention of expressing the head turned inwardly (with its head inside the outline of the body) or outwardly (with its head depicted above the line of its back).

A detailed analysis of the essential characteristics of these representations determines a series of aspects they possess in common and which give them the status of a "thematic group". It equally suggests some hypotheses regarding their technological (orientation), chronological and contextual (thematic associations) character which provide insights into their significance. They do not only express movement and traits of animal ethology, but also form a kind of complex graphic model that is repeatedly imitated.
\end{abstract}

\section{RESUMEN}

Se presenta el estudio de conjunto de las representaciones paleolíticas de zoomorfos con la cabeza vuelta hacia atrás. Su análisis detallado permite enunciar algunas hipótesis de carácter tecnológico (orientación), cronológico y contextual (asociación temática), al tiempo que nos acerca a su significación. Las grafías zoomorfas retrospicientes forman un pequeño grupo característico dentro del repertorio gráfico del arte paleolítico, tratándose de un motivo gráfico que además de reflejar actitudes animales reales debió convertirse, muy posiblemente, en un elemento clave de trasmisión gráfica, debido a su amplia dispersión geográfica.

\section{LABURPENA}

Burua atzera duten zoomorfoen irudikapen paleolitikoen multzoaren inguruko azterketa aurkeztu dugu honekin. Azterketa zehatzari esker, teknologia (orientazioa), kronologia eta testuinguru (lotura tematikoa) arloko hipotesi batzuk adieraz ditzakegu esanahira gerturatzeaz gain. Grafia zoomorfo erretrospizienteek arte paleolitikoaren bilduma grafikoaren baitan talde bereizgarri txikia osatzen dute. Animalien benetako jarrerak islatzeaz gain, seguru asko transmisio grafikoko oinarrizko elementua bihurtuko zen hedapen geografiko handia duelako.

\footnotetext{
(1) Área de Prehistoria. Dpto. Ciencias de la Antigüedad. Universidad de Zaragoza. Facultad de Filosofia y Letras. c/ Pedro Cerbuna 12, 50.009 Zaragoza. albertrisa13@hotmail.es

(2) Dpto. de Prehistoria, Historia Antigua y Arqueología. Universidad de Salamanca. clara85@usal.es

(3) Investigador Postdoctoral "Torres Quevedo". 3D Scanner Patrimonio e Industria. Spin-off Universidad de Zaragoza. Proyecto HAR201127197. manubea@unizar.es
} 


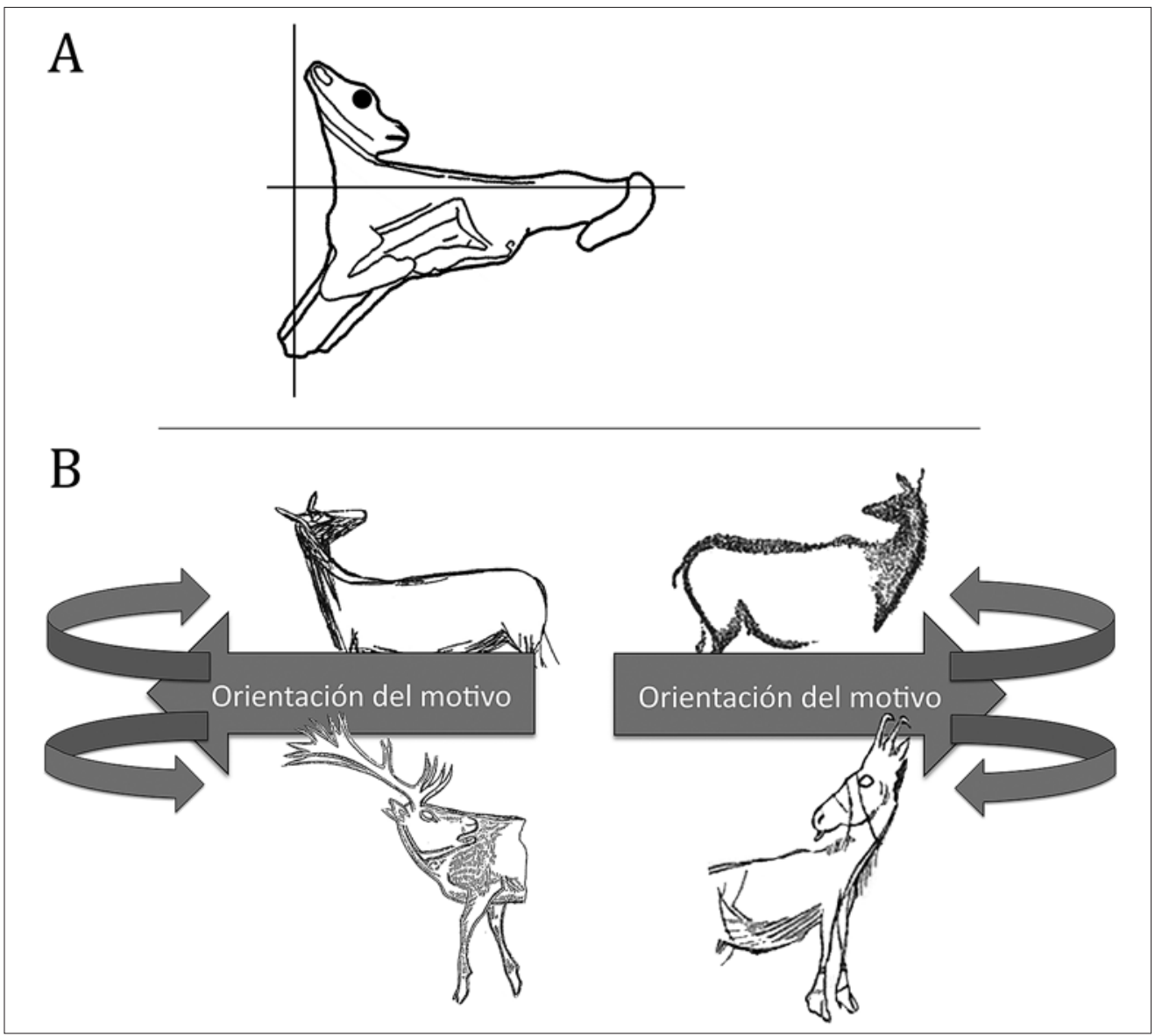

Fig. 1. A. Modelo de motivo retrospiciente con la cabeza girada respecto al eje vertical imaginario que marca la posición frontal de la cabeza. B. Diferentes giros de cabeza según orientación del motivo y lateralidad en el giro. / A. Model of a retrospiscens motif with the head turned with respect to the imaginary vertical axis that marks the frontal position of the head. B. Diferent head turns depending on the the orientation of the motif and the laterality of rotation.

\section{1.- INTRODUCCIÓN}

En el bestiario gráfico paleolítico destaca la presencia, por otra parte inhabitual (ROUSSOT, 1997: 77, fig. 44), de zoomorfos que han sido representados con la cabeza vuelta hacia atrás, definidos como retrospicientes. Son figuras cuya cabeza se vuelve en dirección opuesta al cuerpo, girando de $90^{\circ}$ a $180^{\circ}$ respecto a un eje vertical imaginario que marcaría la posición frontal de la cabeza (figura 1.A). Dicha terminología hace alusión a la actitud móvil del animal y tiene su origen en la iconografía cristiana, donde la figura de un cordero retrospiciente (Agnus Del), muchas veces herido por una lanza o degollado, es interpretada como el sacrificio de Jesucristo por los pecados de los hombres. La comparación entre dicha representación y la primera grafía de caballo descubierta con la cabeza vuelta documentada en Pair-non-Pair (DALEAU, 1896: 245) le valió el epíteto a esta última. Posteriormente se descubrió una cierva representando el mismo movimiento en un panel de la cueva de Covalanas "selon toute probabilité, figurant une horde de Biches surprise par un enemi" (ALCALDE DEL RíO et al., 1911: 16). Sin embargo, transcurrió medio siglo hasta que Leroi-Gourhan sistematizara dicha actitud dentro de la categoría de animación segmentaria (1984: 154, 1992: 266), y otras cuatro décadas hasta que Azéma (2003, 2006: 484, 2010) retomara la investigación de esta forzada posición, si bien analizó únicamente su presencia en las grafías de équidos ${ }^{1}$ del $\mathrm{Pa}$ leolítico francés (2006: 484).

Diversos autores han tratado de explicar a qué se debe esta peculiar manera de representar a los zoomorfos. Por un lado se ha constatado la adaptación al soporte (encuadre) como la causa determinante de la realización de este tipo de grafías (LEROI-GOURHAN, 1984: 154-155, 1992: 267), lo que explicaría ciertas distorsiones anatómicas documentadas (BARANDIARÁN, 1984: 313, 2006: 40 y ss.). Sin embargo, la mayoría de los zoomorfos retrospicientes se representan con la cabeza por encima del cuerpo. Una dinámica gráfica que, desde un punto de vista técnico, requeriría una cierta longitud del espacio gráfico disponible, sobre todo en animales de cuellos largos, como los caballos. Por tanto, la limitación espacial parece únicamente una explicación

${ }^{1}$ Dicha convención (2-1, 2-2 y 2-3 de la tabla 3 en AZÉMA, 2003) se analiza únicamente en relación a las grafías de caballos. El autor señala que en otras áreas geográficas, como la Región Cantábrica, esta animación se halla también en las representaciones de cérvidos (AZÉMA, 2006: nota 4). 
plausible para la realización de cabezas retrospicientes dentro del cuerpo, por lo que no puede admitirse como hipótesis unívoca para el gran número de grafías y morfologías que participan en dicha definición.

Este modelo gráfico también podría responder al reflejo fiel del comportamiento de ciertos animales, tal y como muestra la etología (BANDI et al., 1984; CLOTTES et al., 1994a, 1994b; PAILLET, 1999). Desde dicha disciplina se han interpretado algunas grafías como bisontes lamiéndose (LEROI-GOURHAN, 1971: 431), cervatillos defecando (CAMPS, 1984) o pariendo (BANDI, 1988: 140), ciervos heridos bramando o gimiendo (GONZÁLEZ SAINZ, 2007: 313), ciervas vigilantes ante posibles depredadores cercanos (GORROTXATEGI, 2000: 254; MENÉNDEZ y QUESADA, 2008: 163) o bisontes revolcándose en la tierra (BANDI, 1984: 565). Así, la ejecución de estas grafías respondería a la elección por parte de un grupo humano de plasmar una determinada iconografía, cuyo significado es conocido y compartido por todos los miembros de dicha comunidad.

Más allá de su significado algunos autores han señalado la complejidad de este motivo, al tratarse de un concepto gráfico que trasciende el tipo de soporte, siendo documentado en el ámbito parietal y mueble, sin una variación significativa del tema desarrollado (SAUVET et al., 2008b: 74). De esta manera se ha señalado la capacidad de estas grafías como trasmisores conceptuales de ideas o intercambios a corta y larga distancia entre los grupos humanos paleolíticos (SAUVET et al., 2008: 35; FRITZ et al., 2007: 175), pudiendo actuar como verdaderos marcadores territoriales. La repetición de este tipo de representaciones en el arte paleolítico justifica su valor como modelo iconográfico (GORROTXATEGI, 2000: 254).

El presente estudio tiene como objetivo concretar en el espacio y en el tiempo esta convención gráfica y contrastar sus posibles explicaciones. El conocimiento de la distribución, reinterpretación y de las particularidades de dicho modelo puede acercarnos a las sociedades paleolíticas que las produjeron, a su simbología y a sus formas de transmisión o conceptualización existentes en el pasado.

\section{2.- METODOLOGÍA Y VARIABLES DE ANÁLISIS}

Ambas explicaciones (la etológica y la adaptativa/funcional) deben ser contrastadas, por lo que se ha propuesto un análisis formal y técnico de estas grafías (55 unidades gráficas, vid. anexo) a partir de un conjunto ordenado de 7 variables cualitativas: cronología, espacio gráfico, tema o familia zoomorfa, técnica, tipo de composición/asociación, lateralidad y modo de representación.

Las unidades gráficas que componen el corpus analizado responden a la siguiente descripción: zoomorfo con cabeza girada $180^{\circ}$ sobre su eje. Por tanto se descartan del análisis las grafías de animales que, con el cuerpo de perfil, giran la cabeza hacia el frente $\left(90^{\circ}\right)$, como ocurre en las cabras del hueso de Torre (BARANDIARÁN, 1971: fig. 2) o en la cabra del bloque de la cueva de Abauntz (UTRILLA y MAZO, 1996: 50, fig. 9), pues si bien giran la cabeza no lo hacen hacía atrás en el sentido que aquí nos interesa. Sin embargo, se han incluido grafías con dos cabezas, siempre y cuando una de ellas se oriente hacia atrás (vid. Quinta da Barça, E1).

En primer lugar recogemos la distribución espacial y cronológica. Para subsanar las imprecisiones de las dataciones estilísticas de algunas de las representaciones de los zoomorfos retrospicientes, así como la descontextualización de ciertas piezas de arte mueble, se ha dividido en cuatro los períodos crono-culturales en los que se engloban las grafías del repertorio: el período 1 o Pre-Magdaleniense, en el que se incluye el espacio de tiempo asignado a los tecno-complejos Gravetiense y Solutrense; el período 2, para el Magdaleniense inferior, en el que participan los tecno-complejos Magdaleniense inferior cantábrico y el Magdaleniense III francés; el período 3, para el Magdaleniense medio; y el período 4, equivalente al Magdaleniense superior-final.

El segundo dato que hacemos constar son las especies animales que intervienen en el corpus de figuras recopilado, distinguiendo las familias zoomorfas más representadas (cervidae, bovidae y caprinae). La identificación de las especies es la comúnmente aceptada, aunque hemos identificado como cabras, tal y como apunta Barandiarán (2006: 59), y no como gamuzas las figuras de los propulsores de Bédeilhac y Más d'Azil (CLOTTES, 2001: 57-58).

Como se ha señalado para el arte mueble, la forma y las dimensiones de los soportes condicionan en gran medida las representaciones gráficas (DELPORTE, 1976: 39; LEROI-GOURHAN, 1984: 155). El arte mueble, sobre todo en materia ósea o en asta, dispone siempre de un campo gráfico reducido con respecto al soporte parietal, lo que genera algunas diferencias en las grafías retrospicientes. Por este motivo también hemos cuantificado las unidades muestreadas en función del tipo de soporte en que éstas se ejecutaron: 29 UGs parietales y 26 UGs muebles.

Se recoge igualmente la técnica en la que fueron realizadas estas representaciones. El grabado², la pintura, la técnica mixta (grabado y pintura), el trazo digital, el bajorrelieve, la escultura y el piqueteado.

Debido al mencionado grado de naturalismo y dinamismo de las grafías objeto de estudio hemos recogido los datos referentes a la actitud (dinámica, estática, en alerta o herido) y el carácter cuantitativo de la composición (aislada, doble, triple o en grupo). El recuento de zoomorfos se ha efectuado a partir de la presencia de determinadas partes anatómicas, de modo que aunque los individuos sean incompletos, la presencia de una de

2 Sin tener en cuenta las variantes de las técnicas referidas como el grabado estriado en el que fue realizada la cierva de un omóplato de El Castillo (CORCHÓN, 1996: 317, fig. 73) o el punteado de la cierva retrospiciente de Covalanas (GARCÍA-DíEZ y EGUIZÁBAL, 2003: 45, fig. 12). 
sus partes se contabiliza como una unidad gráfica diferenciada como ocurre en la roca 3 de Quinta da Barca (BAPTISTA, 1999: 12), donde se registra una tercera cabra, aunque sólo se conserve de ésta el cuarto trasero. Para analizar la naturaleza de la composición hemos dividido las asociaciones utilizado la terminología de Barandiarán (2003: 13). Existen dos tipos de asociaciones: 1) homoespecíficas (o intraespecíficas), que son aquellas representaciones en las que las figuras retrospicientes aparecen junto a otra/s de la misma especie en muy clara relación espacial (en un mismo panel o cara de un objeto mobiliar), técnica y estilística; y 2) heterogéneas (o interespecíficas), entendidas como aquellas representaciones en las que las grafías retrospicientes se relacionan con otra $u$ otras de especies distintas. Podría así mismo considerarse un tipo de asociación dudosa en la que las relaciones de las figuras no están claras. Para los dos primeros casos (homoespecíficas o heterogéneas) se ha especificado el número de figuras que participan en las asociaciones: dobles, triples o múltiples (más de tres zoomorfos asociados).

En el análisis participa también la dirección a la que apuntan las cabezas retrospicientes: mirando a izquierda, que equivale al perfil derecho de la figura, y mirando a derecha, que equivale al perfil izquierdo. Esto no es analizable en los zoomorfos realizados en bulto redondo de los propulsores de Bédehilhac y de Mas d'Azil, pues en ellos la visualización del artefacto por parte del observador determina la direccionalidad de la cabeza del zoomorfo, por lo que estos casos no se han contemplado en el recuento. En determinados casos se ha seleccionado la orientación "natural" del animal, como la cabra del canto grabado de Bruniquel (SIEVEKING, 1987a: 94, fig 662) volteando la pieza $180^{\circ}$ a como se suele presentar (figura 2).

Por último hemos establecido una clasificación para el tipo de representación de las cabezas retrospicientes, que pueden hallarse dentro o fuera del cuerpo del animal.

\section{3.- ANÁLISIS DE LOS DATOS}

El corpus de unidades gráficas (UGs) retrospicientes analizado se compone de 55 ejemplares ( vid. anexo). En su distribución crono-cultural destaca la presencia de este concepto gráfico en el Magdaleniense medio (25 UGs) seguido del ciclo gráfico Pre-magdaleniense (16 UGs), Magdaleniense superior-final (10 UGs) y, ya muy alejado, del Magdaleniense inferior (4 UGs) (figura 3).

El cálculo porcentual de las especies zoomorfas/antropomorfas que responden a esta convención gráfica muestra la preferencia de los cérvidos (29\%) y cápridos (25\% incluyendo las grafías de gamuza), mientras que los équidos, el animal cuantitativamente más representado en el arte paleolítico, apenas alcanza el 15\% de la muestra (figura 4 y tabla II). Esta diferencia genérica advierte una elección de las especies gráficas por parte de sus autores, que parece relacionarse de modo directo con el soporte gráfico, puesto que mientras los cérvidos (11 UGs) y los équidos (7 UGs) se priorizan sobre los paneles rupestres, los cápridos (9 UGs), los bóvidos (4 UGs) y los antropomorfos (3 UGs) predominan en los soportes mobiliares. Sin embargo, esta observación está condicionada por la cantidad de la muestra. Al menos en la Región Cantábrica el número de grafías figurativas muebles documentadas con anterioridad al tecnocomplejo Magdaleniense es casi anecdótico ${ }^{3}$, por lo que resulta lógico que el número de las grafías retrospicientes con carácter mobiliar también lo sea. Igualmente en el suroeste de Francia las obras muebles figurativas con atribución estratigráfica segura son escasas (SAUVET et al., 2008: 46). Finalizado el Magdaleniense inferior las cifras se equilibran y el análisis gana peso interpretativo.

Si ampliamos la vista e introducimos el campo gráfico o panel en el análisis se observa la presencia de grafías retrospicientes asociadas (33 UGs), que destacan en el arte parietal (24 UGs) durante el período 1 (Pre-Magdale-

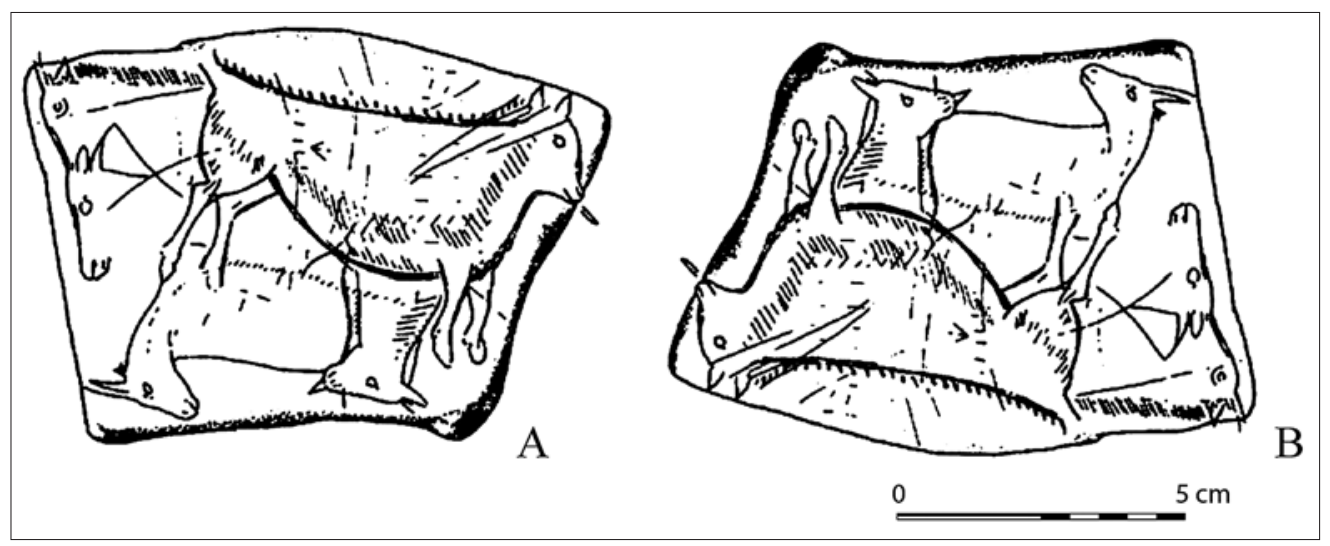

Fig. 2. A. Calco del canto grabado de Bruniquel según la orientación clásica (según SIEVEKING, 1987a: 94, fig. 662). B. Calco con la orientación designada para la lectura propuesta de la cabra retrospiciente. / A. Trace of the engraved pebble from Bruniquel according to the classical orientation (after SIEVEKING, 1987a: 94, fig. 662). B. Trace according to the orientation for the proposed interpretation of the retrospiscens goat.

${ }^{3}$ Los testimonios gráficos figurativos más antiguos proceden de Hornos de la Peña (cuya atribución auriñaciense ha sido recientemente confirmada, TEJERO et al., 2008), de El Castillo (BARANDIARÁN, 1973: 106; CORCHÓN, 1986: 254-255) de Morín (GONZÁLEZ ECHEGARAY y FREEMAN, 1971: 245; BARANDIARÁN, 1973: 147-148; CORCHÓN, 1986: 254-255) y de Antoliñako (AGUIRRE y GONZÁLEZ SAINZ, 2011; GARCÍA-DÍEZ y OCHOA, 2012, 2013). En el Solutrense destaca una pata esculpida sobre un estilete de Bolinkoba, atribuido al Solutrense medio (BARANDIARÁN, 1950: 98 y 103), un caballo grabado sobre hueso hallado en los niveles solutrenses terminales de la cueva de Las Caldas (MENÉNDEZ, 1997: 135; CORCHÓN, 2004: 441) y la escultura de un ave en El Buxu (MENÉNDEZ y OLÁVARRI, 1983). 


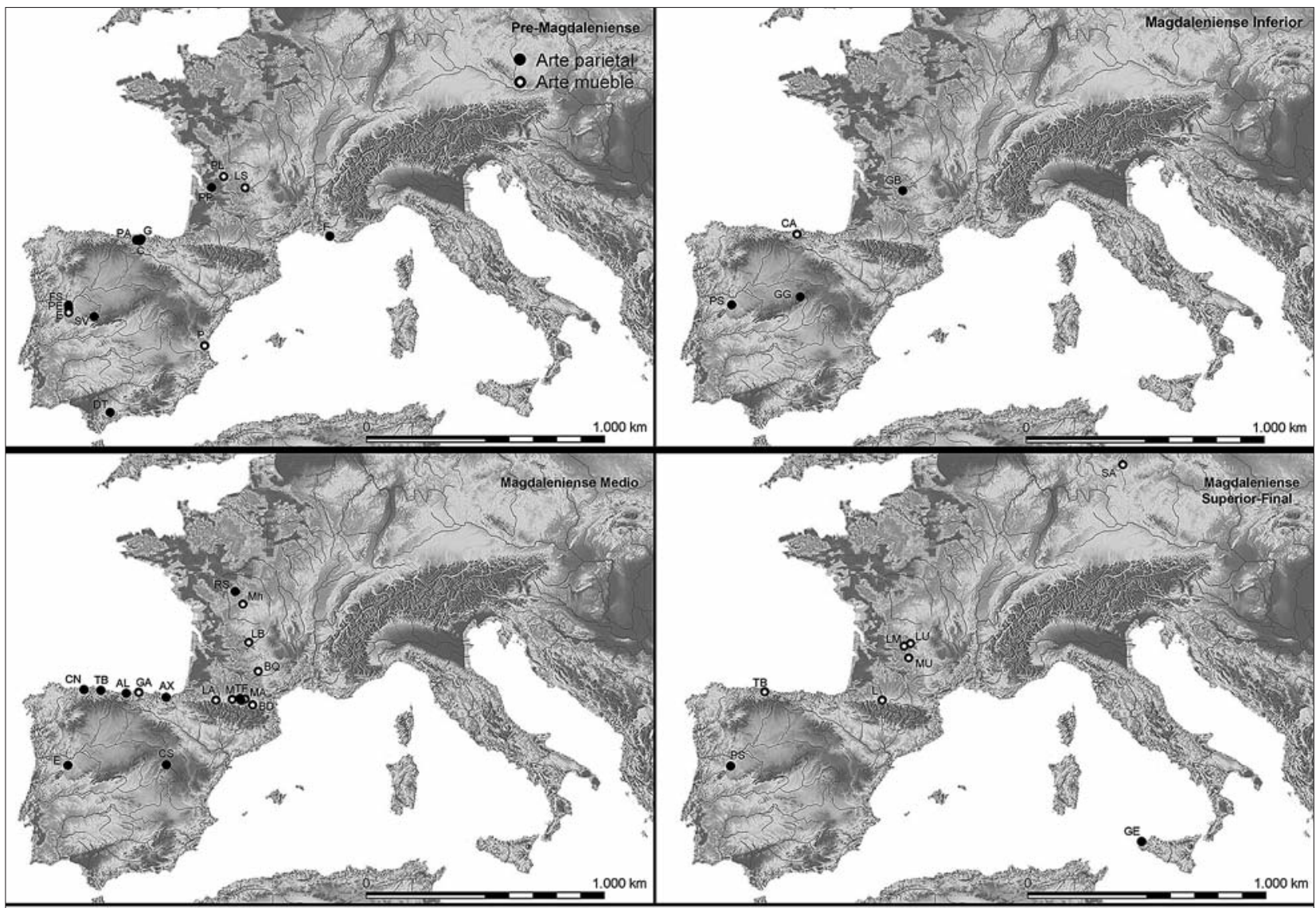

AL - Altamira; AX - Altxerri; BD - Bédei hac; BQ - Bruniquel; C - Covalanas; CA - EL Castillo; CN - Candamo; CS - Los Casares; DT - Doña Trinidad; E - Quinta da Barça; F - Figuier; FS - Fariseu: G - La Garma;
GE - Genovesi; BG - Le Gabillou; GG - La Griega; L - Lortet; LA - Labastide; LB - Laugerie-Basse; LM - La Madelaine; LS - Laussel; LU - Limeuil; M - Montastruc; MA - Mas d'Azil; Mh - La Marche; MU - Murat GE - Genovesi; BG - Le Gabillou; GG - La Griega; L - Lortet; LA - Labastide; LB - Laugerie-Basse; LM - La Madelaine; LS - Laussel; LU - Limeuil; M - Montastruc; MA - Mas d'Azil; Mh - La Marche; MU - Murat;
P - Parpallo; PA - La Pasiega; PE - Penascosa; PL - Placard; PP - Pair-non-Pair, PS - Ribera do Piscos RS - Rocaux-Sociers; SA - Saafeld; SV - Siga Verde; TB - Tito Bustillo; TF - Trois-Frères

Fig. 3. Dispersión de las grafías retrospicientes en el Pre-Magdaleniense, Magdaleniense Inferior, Magdaleniense Medio y Magdaleniense Superior-Final. / Dispersal of the retrospiscens motifs in the Pre-Magdalenian, Lower Magdalenian, Middle Magdalenian and Upper-Final Magdalenian.

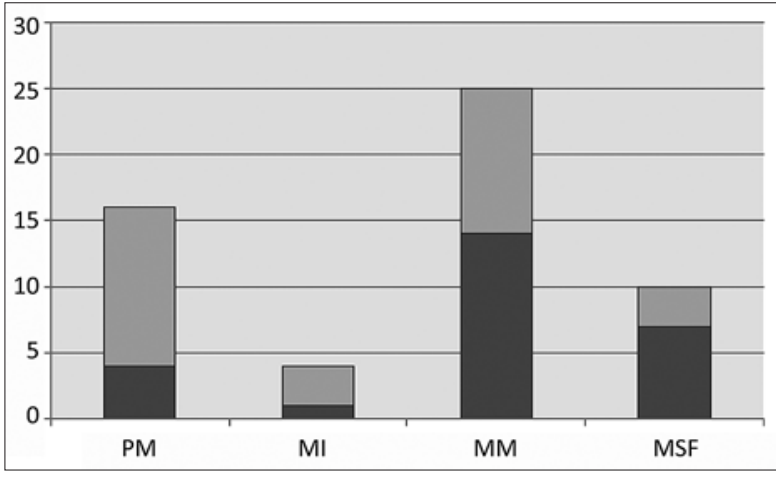

Fig. 4. Distribución de las unidades gráficas documentadas por períodos y en función del soporte gráfico empleado: gris claro (rupestre/parietal) y gris oscuro (mueble). PM) Pre-Magdaleniense; MI) Magdaleniense Inferior; MM) Magdaleniense Medio; MSF) Magdaleniense Superior-Final. / Dispersal of the graphic units documented according to periods and graphic supports used: light grey (rock-art) and dark grey (mobiliar). PM) Pre-Magdalenian; MI) Lower Magdalenian; MM) Middle Magdalenian; MSF) Upper and Final Magdalenian.

niense: 11 UGs), seguidas de las asociaciones aisladas (22 UGs) que son mayoritarias en los dispositivos muebles (17 UGs) durante el Magdaleniense medio (10 UGs). Las asociaciones homoespecíficas presentan un gran número de animales de la misma especie en asociación múltiple, lo cual es poco frecuente al menos en el arte parietal (LEROI-GOURHAN, 1992: 281-287) y dan la idea de grupo o manada. En esta línea sería posible considerar el conjunto de Quinta da Barça como una pequeña manada (LEROI-GOURHAN, 1992: 282), mientras que en otros casos, como en el bastón grabado de Lortet, esta realidad puede responder a la representación de un tipo de animación concreta (UTRILLA y MARTíNEZ BEA, 2005/06: 163). Existen también casos de asociaciones homoespecíficas grupales, como las cabras de Roc-aux-Sociers, los caballos de la Roca 3 de Ribeira de Piscos, las ciervas del panel principal de Covalanas, los renos en Limeuil y los bisontes de Altamira. Este tipo de asociaciones han sido interpretadas como la expresión de comportamientos etológicos ligados a reagrupamientos estacionales (DUBOURG, 1994: 169-170) o como la reproducción de un mismo zoomorfo en movimiento a partir de la multiplicación de su contorno (AZÉMA, 1992: 65-68).

En cuanto a la orientación de las grafías existe un marcado equilibrio (dcha.=26 UGs; izda.=27 UGs) tanto en las unidades parietales como en las mobiliares. Tan sólo dos unidades no han podido ser codificadas bajo este criterio, 
pues se trata de dos grafías talladas sobre un propulsor de asta, de modo que la direccionalidad del zoomorfo depende de su observación y no de su materialidad.

Por último, la mayoría de las grafías zoomorfas retrospicientes se realizan con la cabeza por encima del cuerpo (42 UGs), lo que requiere de cierto espacio gráfico. En ese sentido no se perciben grandes diferencias entre el arte parietal y el mueble (siempre teniendo en cuenta la escasa representatividad de la muestra), salvo en la representación de caballos. La preferencia de los caballos en el arte parietal (7/8) respecto al mobiliar (1/8) puede deberse a la comodidad de espacio que ofrece el soporte parietal, ya que éstos presentan un cuello largo, lo que exige bastante altura para su cómoda realización, como en los caballos de Pairnon-Pair. Los 13 casos en los que la cabeza se localiza dentro del cuerpo parecen haber estado condicionados por el espacio gráfico disponible (LEROI-GOURHAN, 1984: 155) (tabla I), como puede ser el caso del bisonte localizado en el techo de Altamira (AL1), en soportes estrechos como el propulsor de La Madeleine (LM1) o de Trois-Frères (TF3), donde la cabeza del ánade parece un trasunto formal del apéndice del propulsor, en los bastones perforados de Laugerie-Basse (LB1 y LB2) y Lortet (L1) y en el omóplato de Laugerie-Basse (LB3), donde un apéndice del soporte ubica el cuerno de la cabeza retrospiciente de una cabra. Sin embargo, otras grafías procedentes de Tito Bustillo (TB2), Labastide (LA1), Montastruc (M1) y Murat (M1 y M2) se enmarcan en soportes (muebles o parietales) lo suficientemente amplios para que la cabeza pudiese trazarse fuera del cuerpo y no sucede así, por lo que debe admitirse una significación cultural para esta convención, empleada selectivamente por parte de sus autores.

Valorando la observación precedente, es posible que las cabezas retrospicientes realizadas dentro del cuerpo estén representando un tipo de animación diferente: el giro interior del animal al volverse sobre sí mismo. Así existirían dos tipos de giros de cabezas en los zoomorfos retrospicientes: una hacia el interior (con la cabeza representada dentro del cuerpo) y otra hacia el exterior del cuerpo (con la cabeza por encima de la línea dorsal) del zoomorfo representado. Dicha divergencia se observa en zoomorfos que miran en una misma dirección pero que giran la cabeza en sentidos opuestos (figura 1.B). Es posible que en estos casos la cabeza en el interior o exterior del cuerpo del zoomorfo no sea fruto de la casualidad ni de la adaptación a un soporte restringido, sino el resultado de la voluntad del artista por plasmar los distintos giros de la cabeza animal.

Desde este punto de vista, cabe observar que las cabezas retrospicientes de los bisontes estén lamiéndose (LEROI-GOURHAN, 1971: 431) o revolcándose en la tierra para impregnarse de olores (BANDI, 1984: 556-557), siempre se representan en el interior del cuerpo y nunca girando al exterior del perfil observable, por la sencilla razón de que su abultada giba y la brevedad del cuello imposibilitan el movimiento de la cabeza por encima de la línea cérvico-dorsal. Esto podría explicar el hecho de que todas las cabezas retrospicientes de bisontes hayan sido realizadas dentro del cuerpo 4 .

\section{4.- ANÁLISIS DE LOS DATOS Y VALORACIÓN POR PERÍODOS}

\subsection{Período I: Pre-Magdaleniense}

En el ciclo gráfico más antiguo del Paleolítico esta convención casi se muestra exclusiva del soporte parietal (13 UGs) sobre el que han sido representados 1 megaceros (La Garma, G1), 4 ciervos (Siega Verde-SV1, FariseuFs1, Quinta da Barca-E2 y Doña Trinidad-DT1), 3 ciervas (Covalanas-C1, La Pasiega-PA1 y Doña Trinidad-DT2), 4 caballos (Pair-non-Pair-PP1 y PP2, Penascosa-PE2 y Figuier-F1) y 1 uro (Penascosa, PE1). Dicha apreciación no es significativa en cuanto que todos los corpus globales muestran una notable desproporción entre ambos soportes para el período que nos ocupa (vid. supra). Sobre soporte mueble se contabilizan 3 UGs procedentes de Parpalló (1 cabra), Laussel (1 antropomorfo) y Placard (1 gamuza). En cuanto al bestiario gráfico éste responde per-

\begin{tabular}{|l|l|l|l|}
\hline $\begin{array}{l}\text { Yacimiento } \\
\text { Altamira }\end{array}$ & $\begin{array}{l}\text { Zoomorfo } \\
\text { Bisonte }\end{array}$ & $\begin{array}{l}\text { Soporte } \\
\text { Techo parietal }\end{array}$ & $\begin{array}{l}\text { Bibliografía } \\
\text { BREUIL y OBERMAIER, 1984: 84, fig. 44 }\end{array}$ \\
\hline La Madeleine & Bisonte & Propulsor & LEROI-GOURHAN, 1971: 201 \\
\hline Labastide & Bisonte & Plaqueta & FRITZ et al., 2007: 175, fig. 4.9 \\
\hline Mas d'Azil & Bisonte & Plaqueta & FRITZ et al., 2007: 175, fig. 4.10 \\
\hline Laugerie-Basse & Reno & Bastón perforado & SIEVEKING, 1987b: 3, plate 1A \\
\hline Laugerie-Basse & Reno & Bastón perforado & MULLER-KARPE, 1966: tafel 79. 17a \\
\hline Lortet & Ciervo & Bastón perforado & BARANDIARÁN, 2003: 150, fig. 28 \\
\hline Montastruc & Caballo & Plaqueta & SIEVEKING, 1987: plate 97 \\
\hline Ribeira de Piscos & Caballo & Aire libre parietal & BAPTISTA, 1999: 129 \\
\hline Murat & Cierva & Canto & LEMOZI, 1924: 22, fig. 3 \\
\hline Murat & Bisonte & Canto & LEMOZI, 1924: 30, fig. 7 \\
\hline La Garma & Cabra & Espátula & GONZÁLEZ SAINZ, 2003: 207, foto 1 \\
\hline Tito Bustillo & Ciervo & Parietal & BERENGUER, 1994: 135, fig. 107 \\
\hline
\end{tabular}

Tabla 1: Zoomorfos retrospicientes en los que se ha situado la cabeza dentro del cuerpo. / Retrospiscens zoomorphs with the head inside the body.

${ }^{4}$ En otros casos, como en los caballos de Pair-non-Pair y Roc-aux-Sociers, el concepto artístico parece imponerse a la forma del soporte (BROT, 2012: 86, 87-89). 
fectamente a los porcentajes de presencia característicos del período gráfico arcaico (PETROGNANI, 2013; SAUVET y WLODARCZYCK, 2000/01) en el que el caballo se muestra preeminente seguido de las representaciones de cérvidos (cierva, ciervo y megaceros) y de bóvidos (uro y bisonte) (figura 5 y tabla II).

Las grafías retrospicientes registradas para este período (16) suelen participar en composiciones grupales junto a otras unidades gráficas de la misma especie (3 UGs se muestran en asociación homoespecífica) o de especies diferenciadas (6 UGs en asociación intraespecífica). Las restantes 7 UGs se han ejecutado aisladas (4 UGs), formado parejas (1 UG) o su asociación es dudosa (G1 y E2).

La orientación de la cabeza muestra predilección por la orientación izquierda (10 UGs), al tiempo que existe una marcada preferencia por desplazar la cabeza respecto del cuerpo del animal, de modo que ésta se dispone por encima de la línea cérvico-dorsal del cuadrúpedo.

\subsection{Período II: Magdaleniense inferior}

Para el Magdaleniense inferior (Magdaleniense III francés) tan sólo se han documentado 4 UGs procedentes de El Castillo (CA1), Gabillou (GB1) Ribeira de Piscos (PS) y La Griega (GG1). La adscripción crono-cultural de la primera de ellas no presenta dudas ya que se trata de una representación de cierva grabada en trazo múltiple y relleno estriado sobre omóplato, característica del Magdaleniense inferior cantábrico ${ }^{5}$. Esta unidad participa de una composición mayor (una pareja de ciervas y un caballo) girando su cabeza hacia la derecha sin que ésta se adentre en el cuerpo del animal. El tema escogido es característico de las tradiciones gráficas pre-magdalenien-

\begin{tabular}{|l|c|c|c|c|c|c|c|c|c|c|c|c|}
\hline & P/M & CVO & CVA & CAB & BIS & URO & CBR & GMZ & REN & ANTR & OTRS & TOTAL \\
PM & $12 / 4$ & $3 / 1$ & $3 / 0$ & $4 / 0$ & 0 & $1 / 0$ & $0 / 1$ & $0 / 1$ & 0 & $0 / 1$ & $1 / 0$ & $16(29 \%)$ \\
\hline MI & $3 / 1$ & 0 & $0 / 1$ & 0 & 0 & 0 & $1 / 0$ & $1 / 0$ & 0 & 0 & $1 / 0$ & $4(7 \%)$ \\
\hline MM & $11 / 14$ & $2 / 0$ & 0 & $2 / 1$ & $2 / 2$ & 0 & $3 / 5$ & $0 / 1$ & $0 / 2$ & $1 / 2$ & $1 / 1$ & $25(46 \%)$ \\
\hline MSF & $3 / 7$ & $0 / 2$ & $2 / 2$ & $1 / 0$ & $0 / 1$ & $0 / 1$ & 0 & $0 / 1$ & 0 & 0 & 0 & $10(18 \%)$ \\
\hline TOTAL & $29 / 26$ & \multicolumn{2}{|c|}{$16(29 \%)$} & $7(15 \%)$ & $5(9 \%)$ & $2(4 \%)$ & $10(18 \%)$ & $4(7 \%)$ & $2(4 \%)$ & $4(7 \%)$ & $4(7 \%)$ & $\mathbf{5 5}(\mathbf{1 0 0} \%)$ \\
\hline
\end{tabular}

Tabla 2: Distribución temática en los cuatro períodos analizados y porcentajes globales de la muestra teniendo en cuenta el soporte gráfico $\left(P / M^{*}=\right.$ parietal/ mueble; cvo.=ciervo; cva.=cierva; cab.=caballo; bis.=bisonte; cbr.=cabra; gmz.=gamuza; ren.=reno; antr.=antropomorfo; otrs.=otros). / Dispersal of the graphic units documented according to four analised periods and global percentages in sampling, depending on their graphic supports.

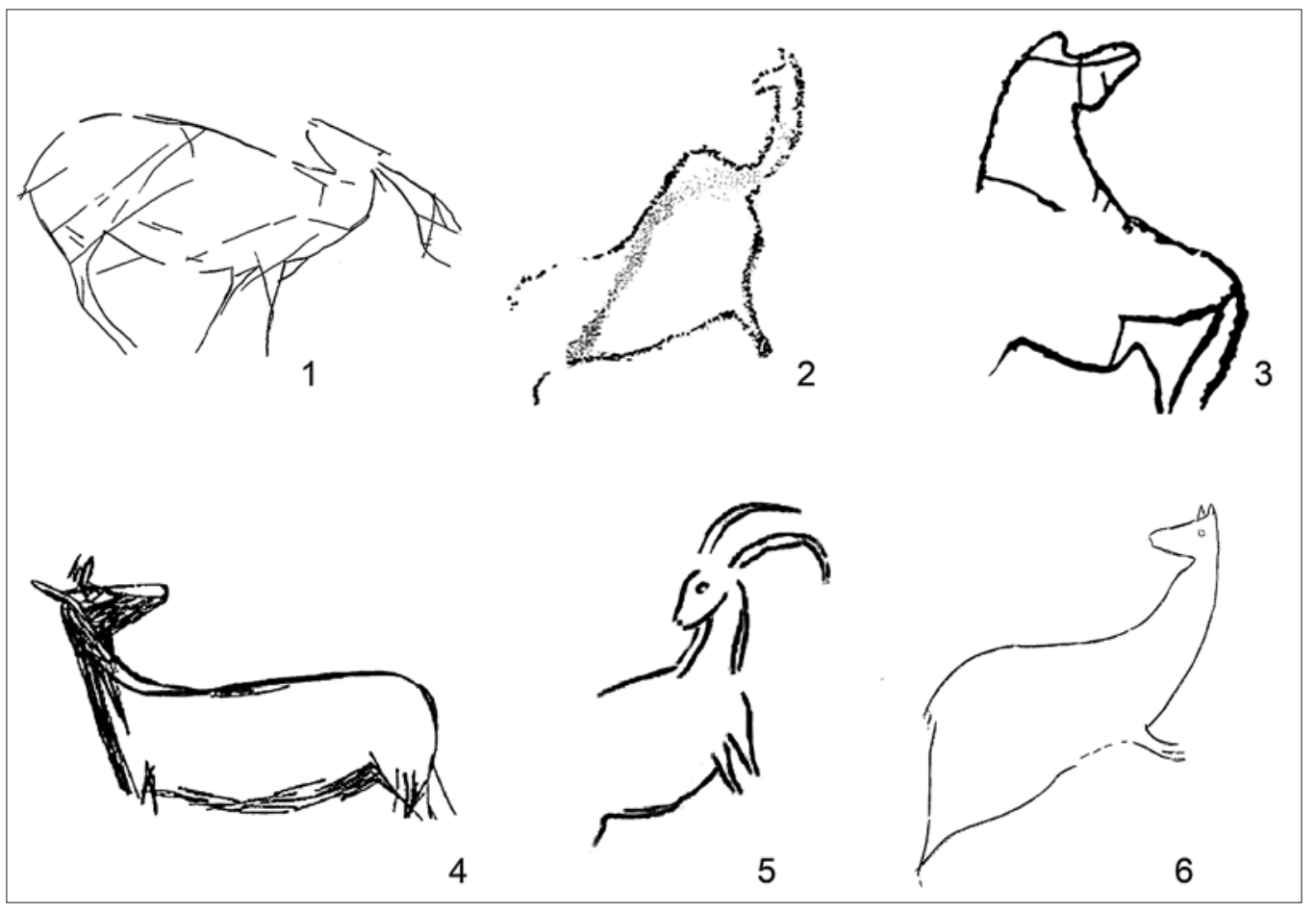

Fig. 5. Ejemplos de representaciones retrospiscientes pertenencientes al Pre-Magdaleniense (1. Parpalló, 2 . La Garma, 3. Penascosa) y Magdaleniense Inferior (4. El Castillo, 5. Gabillou, 6. La Griega). / Examples of the retrospiscens representations in the Pre-Magdalenian (1. Parpalló, 2. La Garma, 3. Penascosa) and Lower Magdalenian (4. El Castillo, 5. Gabillou, 6. La Griega).

${ }^{5}$ La datación directa del omóplato AL5 de Altamira en 14.400‡250 BP (VALLADAS et al., 1992: 69) y más recientemente las del nivel 17 de El Mirón (15.700 190 BP y $14.550 \pm 160$ BP) en el que se han documentado un conjunto de omóplatos grabados (STRAUS y GONZÁLEZ MORALES, 2005: 55) sitúan la ejecución de este modelo gráfico en una horquilla temporal de unos 3000 años, entre ca. 15.700 y 12.534 BP (ca. 18.932 y 14.860 cal BP; OxCal 4.2., IntCal 13). 
ses de la Región Cantábrica y referencia los últimos momentos de "aislamiento" del área respecto a los Pirineos septentrionales. La representación parietal de Gabillou (GB1) se ha identificado como una cabra grabada dispuesta en una composición grupal intraespecífica. Su cabeza se orienta a la izquierda y se ha ejecutado fuera del tronco del zoomorfo. Más complicada se muestra la atribución zoomorfa/antropomorfa ${ }^{6}$ de la grafía de La Griega (GG1), para la que se ha propuesto una cronología Magdaleniense ¿inferior? (fase II documentada en la cavidad) (CORCHÓN, 1997: 161) (figura 5).

\subsection{Período III: Magdaleniense medio}

Durante el tecnocomplejo Magdaleniense medio parece eclosionar esta convención, presente en 25 UGs en ambos tipos de soportes (mueble 14 UGs y parietal 11 UGs) y bajo técnicas gráficas variadas. Los temas implicados son tanto zoomorfos como antropomorfos, señalando además un "ser complejo" de difícil interpretación (BÈGOÜEN y BREUIL, 1958: 51). Las unidades parietales documentadas proceden de Candamo (CN1), Roc-auxSociers (RS1 y RS2), Quinta da Barça (E1), Altxerri A (AX1), Trois-Frères (TF1 y TF2), Altamira (AL1), Tito Bustillo (TB2 y TB3) y Los Casares (CS1). Se trata de dos representaciones de ciervo (CN1 y TB2), dos équidos (RS2 y TB3), dos bisontes (AL1 y TF1), 3 cápridos (RS1, E1, AX1), una representación antropomorfa (CS1) y un ser compuesto (TF2). En ninguno de los casos documentados se trata de grafías aisladas (salvo TF2), sino que es preeminente la composición grupal, seguida de las tríadas y las díadas, destacando la elección de asociaciones intraespecíficas (6 de 11 UGs; 55\%).

Si analizamos la orientación de la cabeza hemos de señalar la predilección por la derecha (7 UGs). Por último, todas las grafías, salvo 2 UGs (AL1 y TB2), disponen la cabeza fuera del cuerpo, por encima de la línea del lomo. AL1 (bisonte) y TB2 (ciervo) son las grafías parietales más antiguas del repertorio que introducen esta variación del giro (ahora interior) de la cabeza del animal. El caso del bisonte responde a la imposibilidad del giro exterior del bovino, sin embargo, en el caso del ciervo es una elección consciente, más cuando se trata de un soporte parietal en el que no existe una constricción de las posibilidades gráficas.

Las grafías retrospicientes muebles adscribibles a este ciclo gráfico (14 UGs) se han identificado como bisontes (2 UGs), cápridos (6 UGs, incluida una gamuza), équidos (1 UGs), renos (2 UGs), antropomorfos o humanos (2 UGs) y un ánade (1 UG). La variabilidad del bestiario se enriquece, destacando la presencia de renos y bisontes, junto a otras especies "raras". Salvo dos casos (BQ1 y LB3), las grafías se han ejecutado aisladas, por lo que no presentan ningún tipo de asociación. La orientación de la cabeza se muestra homogénea (6 UGs a derecha y 6 UGs a izquierda) pero existe una gran variabilidad en relación a la inclusión o exclusión de la cabeza respecto al tronco del animal. Así se cuantifican 6 UGs (LA1, MA2, LB1, LB2, M1 y GA2) en las que la cabeza se dispone dentro del cuerpo del zoomorfo (1 caballo, 2 bisontes, 1 cabra y 2 renos) y otras $8 \cup G$ s en las que ésta se dispone fuera (MA1, BD1, MA3, LB3, BQ1, TF3, Mh1 y Mh 2), por encima de la línea dorsal (4 cabras, 2 antropomorfos, 1 gamuza, y 1 ánade)

La distribución de las figuras retrospicientes asignadas al Magdaleniense medio (desde Asturias hasta el sureste francés) coindice con la señalada por Fritz et al. (2007: 165) como área de intercambio cultural entre grupos, sobre todo durante el 14.500-13.300 BP (17.670$15.996 \mathrm{cal} \mathrm{BP}^{7}$ (SAUVET et al., 2008a: 53), si bien ésta se amplía hacia el sur y sur-este peninsular por la presencia de dicha convención en el valle del Côa (E1) y en el interior peninsular (CS1). Entre las unidades analizadas destaca un grupo temático característico denominado tradicionalmente "le faon aux oiseaux". Se trata de propulsores sobre asta de reno en los que se ha esculpido una representación de cáprido retrospiciente (MA1 y BD1) que gira su cabeza para observar un pájaro que se posa sobre el cuarto trasero de dicho animal. Algunos rasgos como la inexistencia de cornamenta o la presencia de cuentas oculares grandes, redondeadas y huecas -con el objeto de incrustar en ellas algún tipo de pasta de ocre, arcilla o ámbar (FRITZ et al., 2007: 172)- se han relacionado con su carácter juvenil (faon), no exento de críticas (BANDI, 1988: 135). Esta iconografía es común a Bédeilhac y Mas d'Azil, cavidades que se encuentran a apenas $50 \mathrm{~km}$. de distancia; pero también pueden identificarse dentro de este grupo los propulsores sin cabeza de Arudy, Enlène, Isturitz y Labastide realizados en el mismo soporte y técnica. Estas piezas presentan tantas características comunes con sus semejantes retrospicientes que permiten su tratamiento en conjunto (BANDI, 1988; CAMPS, 1984; CLOTTES, 2001; BARANDIARÁN, 2006: 59; FRITZ et al. 2007: 170; SAUVET et al. 2008: 35). Su exclusión en nuestro estudio, por la pérdida de la cabeza, desvirtuaría la muestra de nuestro catálogo. A pesar de la interpretación de estas piezas en los términos referidos, no las hemos incluido en los cálculos globales, puesto que la ausencia de cabeza puede responder a un proceso tafonómico o a la elección por parte de su autor/a de que ésta no fuese representada o se tratase de una pieza móvil o articulada. Por tanto, la existencia de un motivo estereotipado y de su posible reinterpretación gráfica de forma reiterada (SAUVET et al., 2008a: 35) implica, como ya apuntara Bandi (1988: 137), la existencia de un patrimonio simbólico bien establecido, una tradición iconográfica y, posiblemente, una mitología desarrollada regionalmente. Esta misma observación se puede aplicar a los bisontes retrospicientes "lamiéndose el

\footnotetext{
6 "La interpretación del sujeto -una cierva retrospiciente si nos atenemos a la parte superior del cuerpo- puede matizarse como antro-zoomorfo, si tenemos en cuenta el trazado del vientre, extremidades y su estrecho paralelismo con los antropomorfos de los sectores VI y II" (CORCHÓN, 1997: 61). ${ }^{7}$ La datación ha sido calibrada mediante el programa OxCal 4.2., IntCal (REIMER et al., 2013). Fecha de consulta: 05/02/2014.
} 
lomo" documentados en La Madeleine, Mas d'Azil y Labastide (FRITZ et al., 2007: 175, fig. 4), lo que implica un valor más allá de la propia estética y de la reproducción de una actitud animal, al actuar como trasmisores de un conjunto de conceptos formales que se repiten durante el Magdaleniense medio en el registro mueble y parietal del área franco-cantábrica (FRITZ et al., 2007: 175).

\subsection{Período IV: Magdaleniense superior-final}

Para el tecnocomplejo Magdaleniense superior-final se documentan 10 UGs, siete de ellas sobre soporte mueble y tres rupestres. El descenso de la muestra sugiere un cambio en los modos gráficos, muy posiblemente, relacionado con cierto aislamiento de los grupos humanos respecto a momentos anteriores (FRITZ et al., 2007: 165)

Las grafías parietales, procedentes de Genovesi (GE1 y GE2) y Ribeira de Piscos (PS1), se han identificado como ciervas y caballo, respectivamente. Estas representaciones se muestran aisladas (GE2) o participan en composiciones triples (GE1) o grupales (PS1). La orientación de las cabezas es heterogénea (1 UG se dispone a izquierda y otras 2 a derecha) (figura 6).

En el ámbito mueble, para este mismo período gráfico, existe una mayor variabilidad gráfica, conformada por 2 ciervos (L1, Lu1), 2 ciervas (TB1, Mu1), 1 bisonte (LM1), 1 uro (Mu2) y 1 gamuza (Sa1). Se documentan dos modelos de composición característicos: las figuras aisladas
(4 UGs) y grupos (3 UGs). La orientación de la cabeza es predominante hacia la izquierda (3 UGs), situando la cabeza hacia en el interior del tronco del animal en 4 de los 7 casos documentados ( $57 \%$ de la muestra).

\section{5.- RECAPITULACIÓN: NUEVAS PROPUESTAS DE INTERPRETACIÓN}

Merece la pena destacar que una temática tan concreta como la analizada contó con una relativa amplia difusión geográfica durante el período que hemos denominado Pre-Magdaleniense. En ese momento se atiende a una distribución dispersa que encuentra una mayor concentración básicamente en tres núcleos diferenciados (valle del Duero, costa Cantábrica y GaronaVézère), aunque se deben destacar tres ejemplos aislados (Figuier, Parpalló y Doña Trinidad), todos ellos en zonas de proximidad costera mediterránea.

Esa relativa amplia difusión del tema del animal retrospisciente parece caer, sin embargo, en el olvido a comienzos del Magdaleniense, documentándose tan sólo cuatro casos, tres de ellos localizados en los mismos núcleos geográficos que concentraban la mayor cantidad de ejemplos en el período anterior.

Es durante el Magdaleniense medio cuando se produce una verdadera explosión en el número de representaciones retorspiscientes y de yacimientos en los que se localizan. Con todo, es posible observar una concentra-

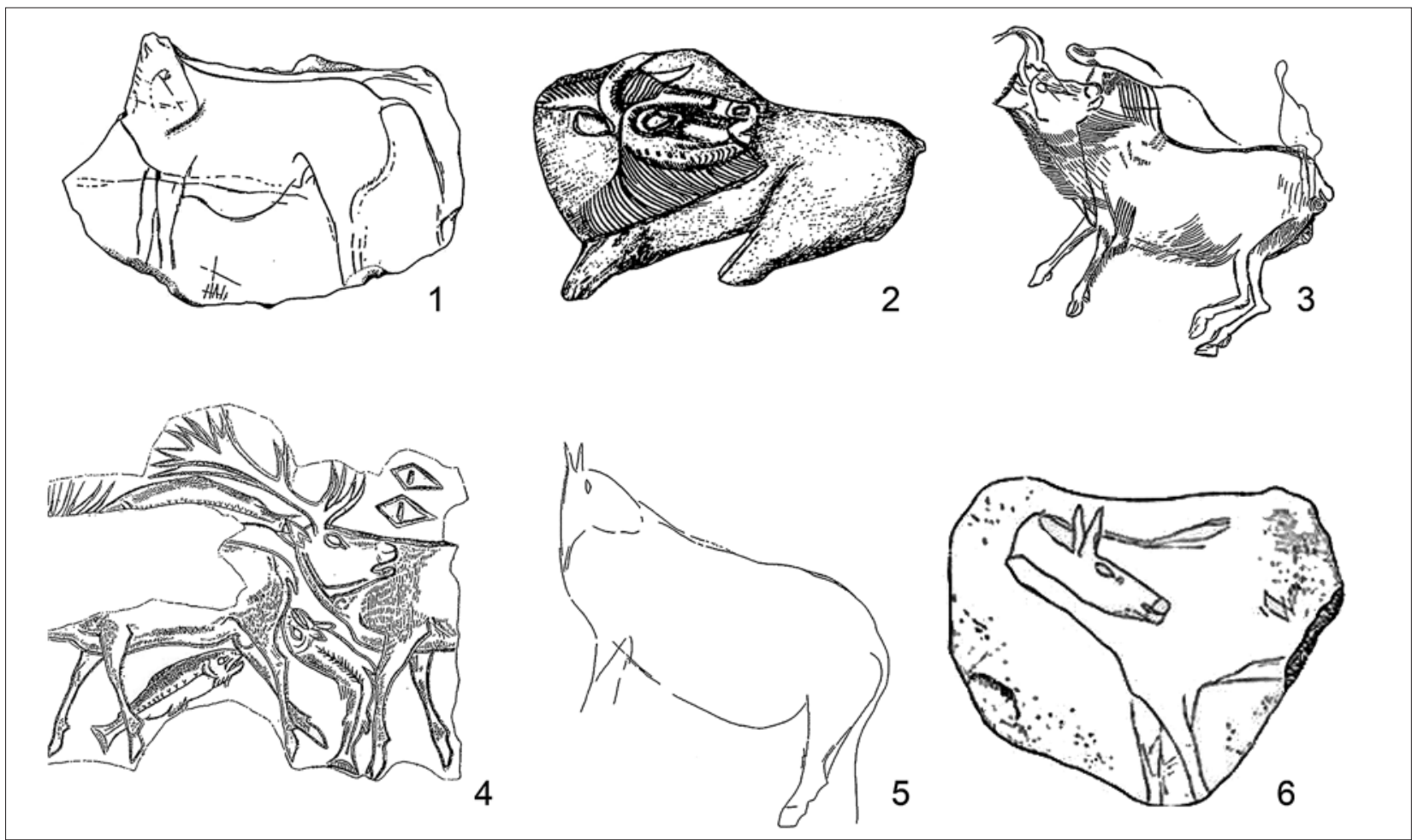

Fig. 6. Ejemplos de representaciones retrospiscientes pertenencientes al Magdaleniense Medio (1. Montastruc, 2. La Madeleine, Trois-Frères) y Magdaleniense Superior-Final (4. Lortet, 5. Ribeira do Piscos, 6. Murat). / Examples of the retrospiscens representations in the Middle Magdalenian (1. Montastruc, 2. La Madeleine, Trois-Frères) and Upper-Final Magdalenian (4. Lortet, 5. Ribeira do Piscos, 6. Murat). 
ción de la temática en dos grandes áreas: costa Cantábrica y Pirineos franceses, con una interesante distribución lineal hacia el Norte con casos aislados en el Lot, Vézère, Vienne y Creusse.

Llama fuertemente la atención la marcada división geográfica de las figuraciones rupestres localizadas esencialmente en yacimientos ibéricos frente a las muebles que se diseminan por los Pirineos franceses y la zona centronorte de Francia. Es precisamente la representación de retrospiscientes en soportes muebles la que más y mejor se perpetúa en el Magdaleniense superior-final, encontrando un pequeño reducto en el Périgord que da continuidad a la tradición de la temática retrospisciente en los mismos soportes que en el período inmediatamente anterior.

Esa dualidad geográfica se constata a nivel global para las representaciones de retrospiscientes en cuanto a la técnica de realización, aspecto determinado por la tipología del soporte (rupestre/mueble). Así, obviando el grabado (la técnica más extendida geográfica y cronológicamente para la temática que nos ocupa), se observa una evidente preferencia por la pintura en la Región Cantábrica, mientras que la escultura aparece de forma exclusiva asociada al componente geográfico pirenaico francés (figura 7).

El análisis de conjunto de representaciones zoomorfas retrospicientes en el arte paleolítico europeo permite evi- denciar una serie de características comunes que dan a estas grafías la cualidad de "grupo temático". Las especies que participan mayoritariamente en este grupo son cérvidos (29\%) y cápridos (25\%, incluyendo las gamuzas), y destacando la escasa presencia del caballo (15\%), el primer zoomorfo del bestiario paleolítico europeo. Predomina su representación en asociaciones grupales (sobre todo homoespecíficas) en paneles rupestres, lo que implica un concepto de narrativa en las composiciones y la posibilidad de que éstas fueran visibilizadas por un número elevado de personas (por oposición al arte mueble, cuyo grado de visibilización es mucho más reducido y depende de la interacción directa entre personas o grupos humanos). En ocasiones estas grafías aparecen condicionadas por el tamaño o la forma del soporte. Sin embargo, en otros casos el naturalismo de los motivos permite identificar en ellos una actitud real de los animales, representada de forma clara, llegando incluso al uso de diversas fórmulas gráficas en función de la animación plasmada. Así se han identificado diferentes giros (interior/exterior) de la cabeza que se reproducen al situar ésta en la parte superior-externa del cuerpo (giro exterior) o en el interior del contorno del tronco del animal (giro interior). Sea como fuere la elección de su reproducción sobre un soporte mueble o parietal debió constituir un modelo gráfico común que, en un momento determinado del Paleolítico

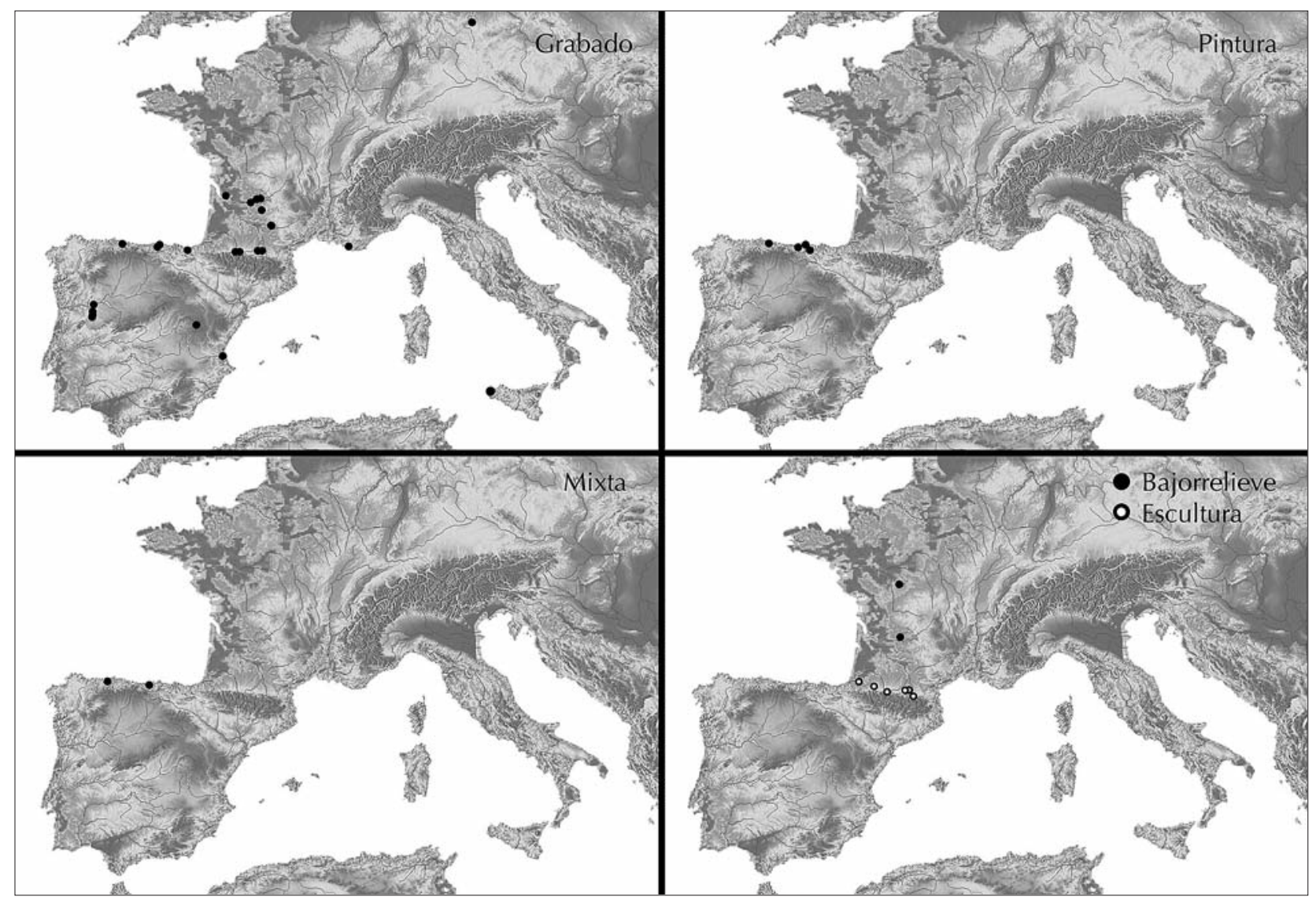

Fig. 7. Distribución de los casos analizados en el estudio según la técnica de ejecución empleada. / Dispersal of the analised cases in the study according to its execution technique. 
superior medio-final, goza de una amplia difusión geográfica, documentándose desde el Ariège hasta la Cornisa Cantábrica, con algunos ejemplos puntuales en la zona centro de la Península Ibérica, Norte de Europa (Saalfeld) y Sicilia (Genovesi).

Todo ello implica un conocimiento preciso del bestiario, de las posibles asociaciones temáticas, integrando una narrativa activa del hecho gráfico. Curiosamente, las representaciones retrospicientes mayoritarias (salvo en el caso de los antropomorfos) coinciden con los especímenes consumidos (cérvidos y cápridos), por lo que el conocimiento etológico de los mismos debió de ser observado y transmitido. La cotidianeidad de las manadas de ciervos y cápridos respecto a los lugares de hábitat favorecería su representación. Basta recordar que numerosas composiciones en las que se documenta una grafía retrospiciente responden, al menos en un primer momento (períodos 1 y 2), a situaciones de alerta o huida (C1, TB2, R1, R2, Lu1, L1, G1) con la presencia de zoomorfos heridos (CN1, F1).

El inhabitual tema de los retrospiscientes se diluye durante las fases finales del Magdaleniense. Ante los últimos artistas paleolíticos aparece ya un horizonte de cambio, y para adaptarse a éste ya no necesitaban mirar atrás.

\section{BIBLIOGRAFÍA}

\section{AGUIRRE, M. y GONZÁLEZ SÁINZ, C.}

2011 Canto con grabado figurativo del Gravetiense de Antoliñako koba (Gautegiz-Arteaga, Bizkaia). Implicaciones en la caracterización de las primeras etapas de la actividad gráfica en la región Cantábrica. Kobie 30, 43-61.

AIRVAUX, J.

2001 L'art préhistorique du Poitou-Charentes. Sculptures et gravures des temps glaciaires. La Maison des Roches. Paris.

ALCALDE DEL RÍO, H.; BREUIL, H. y SIERRA, L.

1911 Les cavernes de la région cantabrique (Espagne). Impr. Vve. A. Chêne. Mónaco.

ALCOLEA, J. J. y BALBíN, R.

2006 Arte paleolítico al aire libre. El yacimiento rupestre de Siega Verde, Salamanca. Arqueología en Castilla y León. Memorias, 16. Junta de Castilla y León.

ALTUNA, J. y APELLÁNIZ, J. M.

1976 Las figuras rupestres paleolíticas de la cueva de Altxerri (Guipúzcoa). Munibe, 28 (1-3), 1-241.

AZÈMA, M.

1992 La représentation du mouvement dans l'art animalier paléolithique des Pyrénées. Bulletin de la Société Préhistorique Ariège-Pyrénées XLVII, 19-76.

2003 La représentation du mouvement dans l'art pariétal francais. Approche éthologique du bestiaire. Thèse de Doctorat, 3 vols. Université de Provence. Aix-Marseille I. Marseille.
2006 La représentation du mouvement au Paléolithique supérieur. Apport du comparatisme éthographique à l'interprétation de l'art pariétal. Bulletin de la Société Préhistorique Française 103 (3), 479-505.

2010 L'Art des Cavernes en action. Tome 2: les animaux figures, animation et mouvement, l'illusion de la vie. Éditions Errance. Paris.

BALBÍN, R.; ALCOLEA, J. J. y GONZÁLEZ PEREDA, M. A.

2003 El macizo de Ardines, un lugar mayor del arte paleolítico europeo. In: Balbín R. y Bueno, P. (eds.): El arte prehistórico desde los inicios del siglo XXI. Primer Symposium Internacional de Arte Prehistórico de Ribadesella. Asociación Cultural de Amigos de Ribadesella.

BANDI, H. G.

1984 Contribution de la zoologie et de l'éthologie a l'interprétation de l'art des peuples chasseurs préhistoriques. L'Anthropologie 88 (4), 563-571.

1988 "Mise bas et non défécation. Nouvelle interprétation de trois propulseurs magdaléniens sur des bases zoologiques, éthologiques et symboliques. Espacio, Tiempo y Forma, Serie I, t. 1, 133-147.

BANDI, H.G.; HUBER, X; SAUTER, M-R. y SITTER, B

1984 La contribution de la zoologie et de l'éthologie à l'interprétation de l'art des peuples chasseurs préhistoriques. In: 3e Colloque de la Société Suisse des Sciences Humaines. Éditions Universitaires. Fribourg

\section{BARANDIARÁN, I.}

1971 Hueso con grabados paleolíticos, en Torre (Oyarzun, Guipúzcoa). Munibe, 23 (1), 37-69.

1973 Arte mueble del Paleolítico cantábrico. Monografías arqueológicas, 14. Zaragoza.

1984 Utilización del espacio y proceso gráfico en el arte mueble paleolítico. In: Scripta Praehistorica Francisco Jorda Oblata: 113-161. Acta Salmanticensia. Salamanca.

2003 Grupos homoespecíficos en el imaginario mobiliar magdaleniense. Retratos de familia y cuadros de género. Victoria: Veleia series minor $n .{ }^{\circ} 21$. Universidad del País Vasco.

2006 Imágenes y adornos en el arte portátil paleolítico. Ariel. Barcelona.

\section{BARANDIARÁN, J. M.}

1950 Bolinkoba y otros yacimientos prehistóricos de la Sierra de Amboto. In: J.M. BARANDIARÁN (ed.): Obras completas de José Miguel de Barandiarán. La Gran Enciclopedia Vasca. Bilbao.

BAPTISTA, M.

1999 No tempo sem tempo. A arte dos caçadores paleolíticos do Vale do Côa. Com uma perspectiva dos ciclos rupestres pós-glaciares. Vila Nova de Foz Côa. Parque Arqueológico Vale do Côa.

2009 O paradigma perdido. O Vale do Côa e a Arte Paleolítica de ArLivre em Portugal. Afrontamento. Lisboa.

BÉGOUËN, R. y BREUIL, H.

1958 Les cavernes du Volp. Trois-Frères. Tuc d'Audoubert. Arts et Métiers Graphiques. Paris. 
BOSINSKI, G.

1982 Die Kunst der Eiszeit in Deutschand und in der Schweiz. Rudolf Habelt GmbH. Bonn.

\section{BERENGUER, M.}

1994 Prehistoric cave art in northern Spain. Asturias (Arte Prehistórico en cuevas del norte de España. Asturias). Frente de Afirmación Hispanista. México.

BREUIL, $\mathrm{H}$. y OBERMAIER, $\mathrm{H}$.

1984 La cueva de Altamira en Santillana del Mar. Ediciones El Viso. Santander.

BROT, J.

2012 L'utilisation des reliefs naturels dans l'art pariétal paléolithique. In: CLOTTES J. (dir.): L'art pléistocène dans le monde / Pleistocene art of the world / Arte pleistoceno en el mundo, Actes du Congrès IFRAO, Tarascon-sur-Ariège, septembre 2010, Symposium «Art pléistocène en Europe». Préhistoire, Art et Sociétés, Bulletin de la Société Préhistorique Ariège-Pyrénées, LXV-LXVI, 2010-2011, CD: pp. 75-91.

CABRÉ, J.

1940-41 Figuras antropomorfas de la cueva de los Casares (Guadalajara). Archivo Español de Arqueología, 14, 81-97.

CANTALEJO, P.; MAURA, R.; ESPEJO, M. M.; RAMOS, J. F.; MEDIANERO, J.; ARANDA, A.; DURÁN, J. J.

2006 La cueva de Ardales: Arte rupestre y ocupación en el Paleolítico superior. Estudios, 1985-2005. Diputación de Málaga. CEDMA

CAMPS, G.

1984 La défécation dans l'art paléolithique In: BANDI, H-G: HUBER, X; SAUTER, M-R. y SITTER, B. (Coords.): La contribution de la zoologie et de l'éthologie a l'interprétation de l'art des peuples chausseurs préhistoriques: 251-261. Éditions Universitaires. Fribourg.

CHEYNIER, A. y BREUIL, $\mathrm{H}$.

1963 La caverne de Pair-non-Pair, Gironde. Fouilles de François Daleau. Société archéologique de Bordeaux, Documents d'Aquitaine III, Bordeaux.

\section{CLOTTES, J.}

2001 Le thème mythique du faon à l'oiseau dans le Magdalénien pyrénéen. Bulletin de la Société Préhistorique AriègePyrénées LVI, 53-62.

CLOTTES, J.; GARNER, M. y MAURY, G.

1994a Magdalenian bison in the caves of the Ariège. Rock Art Research 11, 58-70.

1994b Bisons magdaléniens des cavernes ariégeoises. Bulletin de la Société Préhistorique Ariège-Pyrénées 49, 15-49.

\section{CLOTTES, J; DUPORT, L; FERUGLIO, V. y LE GUILLOU, Y.}

2010 La grotte du Placard à Vilhonneur (Charente) (Fouilles 1990-1995). In: Buisson-Catil, J y Primault, J. (coord.): Préhistoire entre Vienne et Charente. Hommes et sociétés du
Paléolithique. Association des Publications Chauvinoises. Mémoire XXXVIII, 345-358.

\section{CORCHÓN, S}

1986 El Arte mueble Paleolítico Cantábrico: contexto y análisis interno. Centro de Investigación y Museo de Altamira, Monografías, n. ${ }^{\circ}$ 16. Ministerio de Cultura. Madrid.

1996 Reflexiones acerca de la cronología del Magdaleniense cantábrico. Las dataciones 14C de la Cueva de Las Caldas (Asturias. España). Zephyrus XLVIII: 3-19.

1997 La cueva de La Griega de Pedraza (Segovia). Memorias de la Junta de Castilla y León. Consejería de Educación y Cultura. Zamora.

DALEAU, F.

1896 Les gravures sur rocher de la caverne de Pair-non-Pair. Société Archéologique de Bordeaux, 21: 235-249.

DELLUC, B y DELLUC, G.

2006 Une grotte ornée. In: LENOIR, M.; ROUSSOT, A.; DELLUC, B.: DELLUC, G.; MARTINEZ, M.; LOISEAU, S. \& MÉMOIRE, N. (dir.): La grotte de Pair-non-Pair, à Prignacet-Marcamps (Gironde): 23-46. Société Archéologique de Bourdeaux et le Conseil général de la Gironde. Bordeaux.

DELPORTE, $\mathrm{H}$.

1976 Typologie et technologie de l'art paléolithique mobilier. In: ABRAMOVA, Z. y GRAZIOSI, P. (eds.): Les courants stylistiques dans l'art mobilier au paléolithique supérieur: 3753. IXe Congrès de la UISPP. Nice.

1990 L'image des animaux dans l'art préhistorique. Édition Picard. Paris.

\section{DUBOURG, C.}

1994 Les expressions de la saisonnalité dans les arts paléolithiques les arts sur support lithique du Bassin d'Aquitaine. Bulletin de la Société Préhistorique Ariège-Pyrénées 49 145-189.

DUHARD, J-P.

1996 Réalisme de l'image masculine paléolithique. Jérôme MiIlon. Grenoble.

FRITZ, C.; TOSELLO, G. y SAUVET, G.

2007 Groupes ethniques, territoires, échanges: La «notion de frontière» dans l'art magdalénien. In: N. CAZALS, J. GONZÁLEZ URQUIJO y X. TERRADAS (eds.): Frontières naturelles et frontières culturelles dans les Pyrénées préhistoriques (Actas de la Table Ronde Tarascon / sur / Ariège, Mars 2004) Monografías del IIIPC: 164-181. Publican ediciones. Santander.

GARATE, D

2010 Las ciervas punteadas en las cuevas del Paleolítico. Una expresión propia de la Cornisa Cantábrica. Munibe, suplemento 33. Donosti-San Sebastián.

\section{GARCÍA-DÍEZ, M. y OCHOA, B.}

2012 Implicaciones en la secuenciación cronológica rupestre del grafismo figurativo mueble gravetiense peninsular. Veleia 29, 359-372. 
2013 Caracterización del grafismo mueble figurativo Gravetiense en la Península lbérica. In: DE LAS HERAS, C. LASHERAS, J. A.; ARRIZABALAGA, A. y DE LA RASILLA, M. (coords.): Pensando el Gravetiense: nuevos datos para I región cantábrica en su contexto peninsular y pirenaico (Santillana del Mar, Cantabria, 20-22 de octubre de 2011): 633-643. Monografías, 23. Museo Nacional y Centro de Investigación de Altamira. Santander.

GARCÍA DÍEZ, M. y EGUIZÁBAL, J.

2003 Covalanas: el grafismo rupestre y la definición de territorios gráficos en el paleolítico cantábrico. Excmo. Ayto. Ramales de la Victoria. Santander.

\section{GAUSSEN, J.}

1964 La grotte ornée de Gabillou (Près Mussidan, Dordogne). Delmas. Bordeaux.

\section{GONZÁLEZ ECHEGARAY, J. y FREEMAN, L.}

1971 Cueva Morín. Publicaciones del Patronato de las cuevas prehistóricas de la provincia de Santander, VI. Santander.

\section{GONZÁLEZ SAINZ, C.}

1993 En torno a los paralelos entre el arte mobiliar y el rupestre. Veleia 10, 39-56.

2003 "El conjunto parietal de la galería inferior de La Garma (Omoño, Cantabria). Avance a su organización interna". In: BALBÍN, R. de \& BUENO RAMíREZ, P. (eds.): El Arte Prehistórico desde los inicios del siglo XXI: 201-222. Primer Symposium Internacional de Arte Prehistórico de Ribadesella (octubre, 2002).

2007 El tema del «ciervo herido» en el arte parietal paleolítico de la región cantábrica. Evaluación e iconografía. Veleia 24-25, 305-327.

\section{GORROTXATEGI, A}

2000 Arte paleolítico parietal de Bizkaia. Kobie, Anejo 2. Bilbao.

\section{GUTIÉRREZ CUENCA, E; MONTES BARQUÍN, R. y MORLOTE EXPÓSITO, J. M.}

2004 Guía para conocer la cueva de Covalanas. Un santuario rupestre paleolítico en el Alto Asón. Consejería de Cultura, Turismo y Deporte. Cantabria.

\section{HERNÁNDEZ PACHECO, E.}

1919 La caverna de le Peña de Candamo (Asturias). Comisión de Investigaciones Paleontológicas y Prehistóricas. Memoria número 24. Museo Nacional de Ciencias Naturales. Madrid.

IAKOVLEVA, L. y PINÇON, G.

1997 La frise sculptée du Roc-aux-Sorciers, Réunion des musées nationaux. Réunion des Musées Nationaux. Paris.

\section{LEROI-GOURHAN, A}

1971 Préhistoire de l'Art Occidental. Éditions d'Art Lucien Mazenod. Paris.

1984 Arte y grafismo en la Europa prehistórica. Istmo. Madrid.

1992 L'art pariétal. Langage de la préhistoire. Éditions Jérôme Million. Grenoble.
LEMOZI, A

1924 Fouilles dans l'abri sous roche de Murat, Commune de Rocamadour (Lot). Bulletin de la Société préhistorique française 21 (1), 17-58

\section{MENÉNDEZ, M.}

1997 Historiografía y novedades del arte mueble paleolítico en la Península Ibérica. Espacio, Tiempo y Forma, Serie I, t.10, 129-173.

MENÉNDEZ, M. y OLÁVARRI, E.

1983 Una pieza singular de arte mueble de la Cueva del Buxu (Asturias). Homenaje al Prof. Martín Almagro Basch, 1: 319-329.

MENÉNDEZ, M. y QUESADA, J. M.

2008 Artistas y cazadores de ciervos. El papel del ciervo en el arte y la caza del Paleolítico superior cantábrico. Espacio, Tiempo y Forma. Serie I. Nueva época. Prehistoria y Arqueología, 1, 155-166.

MÜLLER-KARPE, H.

1982 Historia de la Edad de Piedra. Gredos. Madrid.

\section{PAILLET, P.}

1999 Le bison dans les arts magdaléniens du Périgord. XXXIIIe supplément à Gallia Préhistoire. CNRS Éditions. Paris.

PALMA DI CESNOLA, A.

1993 II Paleolitico Superiore in Italia. Introduzione allo studio. Garlatti e Razzai Editori. Firenze.

\section{PETROGNANI, S.}

2013 Le style dans tous ses état!. In: Mª.A. MEDINA \& A.J. ROMERO (coods.): Mensajes del Pasado. Manifestaciones gráficas de las sociedades prehistóricas: 25-26. III Encuentro Internacional de doctorandos y postdoctorandos, El Arte de las Sociedades Prehistóricas (Nerja). Fundación Servicios Cueva de Nerja. Córdoba.

REIMER P. J.; BARD, E.; BAYLISS, A.; BECK, J.W.; BLACKWELL, P.G.; BRONK RAMSEY, C.; GROOTES, P.M.: GUILDERSON, T.P.; HAFLIDASON, H.; HAJDAS, I.; HATTŽ, C.; HEATON, T.J.; HOFFMANN, D.L.; HOGG, A.G.; HUGHEN, K.A.; KAISER, K.F.; KROMER, B.; MANNING, S.W.; NIU, M.; REIMER, R.W.; RICHARDS, D.A.; SCOTT, E.M.; SOUTHON, J.R.; STAFF, R.A.; TURNEY, C.S.M. y VAN DER PLICHT, J.

2013 IntCal13 and Marine13 Radiocarbon Age Calibration Curves 0-50,000 Years cal BP. Radiocarbon, 55 (4), 1869-1887.

\section{ROUSSOT, A}

1997 L'art préhistorique. Éditions Sud Ouest. Bordeaux.

\section{SANCHIDRIÁN, J. L.}

2001 Manual de arte prehistórico. Ariel. Barcelona.

\section{SAUVET, G}

1993 La composition et l'espace orné. In: L'art pariétal paléolithique. Techniques et méthodes d'étude: 297-310. Ministère de l'Enseignement supérieur et de la Recherche. Paris. 
2005-06 La latéralisation des figures animales dans les arts rupestres: un exemple de toposensitivité. Munibe 57 (3): 79-93.

\section{SAUVET, G, y WLODARCZYCK, A.}

2000-01 L'art pariétal, miroir des sociétés paléolithiques. Zephyrus 53-54, 217-240.

SAUVET, G.; FORTEA, J.; FRITZ, C. y TOSELLO, G.

2008a Crónica de los intercambios entre los grupos humanos paleolíticos. La contribución del arte para el periodo 20000-12000 años BP. Zephyrus LXI, 33-59

2008b Echanges culturels entre groupes humains paléolithiques entre 20.000 et 12.000 BP. Bulletin de la Société Préhistorique de l'Ariège-Pyrenées LXIII, 73-92.

\section{SIEVEKING, A}

1987a A Catalogue of Paleolithic Art in the British Museum. The Trustees of the British Museum by British Museum Publications. London.

1987b Engraved Magdalenian Plaquettes. A regional and stylistic analysis of Stone, bone and antler plaquettes from Upper Palaeolithic site in France and Cantabric Spain BAR International Series 369. Archaeopress. Oxford.

1988 Animaux en action: un groupe de plaquettes gravées à identité thématique appartenant au Magdalénien tardif. L'Athropologie 92 (1), 41-51.

\section{STRAUS, L. G. y GONZÁLEZ MORALES, M.}

2005 El Magdaleniense de la cueva del Mirón (Ramalas de la victoria, España): observaciones preliminares. In: $\mathrm{BICHO}$ N. (ed): O Paleolítico. Actos do IV Congresso de Arqueología Peninsular: 49-62. Universidade do Algarve. Promontoria Monográfica. Faro.

\section{TEJERO J. M.; CACHO C. y BERNALDO DE QUIRÓS, F.}

2008 Arte mueble en el Auriñaciense cantábrico. Nuevas aportaciones a la contextualización del frontal grabado de la Cueva de Hornos de la Peña (San Felices de Buelna, Cantabria). Trabajos de Prehistoria 65(1), 115-123.

\section{TOSELLO, G}

2003 Pierres gravées du Périgord magdalénien. Art, symboles, territoires. XXXVe supplément à Gallia Préhistoire. C.N.R.S. Éditions. Paris.

\section{UTRILLA, P. y MARTÍNEZ BEA, M.}

2005-06 La captura del ciervo vivo en el arte prehistórico. Munibe 57 vol. III, 161-178.

UTRILLA, P. y MAZO, C

1996 Arte mueble sobre soporte lítico de la cueva de Abauntz. Su aportación a los estilos del Magdaleniense Tardío. Complutum 6 (I), 41-62.

UTRILLA, P.; MAZO, C.; SOPENA, M.C.; DOMINGO, R. y NAGORE, O.

2004 L'art mobilier sur pierre du versant sud des Pyrénées: les blocs gravés de la grotte d'Abauntz. In: LEJEUNE, M. y WELTE, A.-C. (eds.): L'art du Paléolithique supérieur, (Colloque UISPP, Lieja 2001). ERAUL 107, 199-218.
UTRILLA, P.; MAZO, C.; SOPENA, M.C.; MARTINEZ-BEA, M. y DOMINGO, R.

2009 A Palaeolithic map from 13.600 calBP: engraved stone blocks from the Late Magdalenian in Abauntz Cave (Navarra, Spain). Journal of Human Evolution 57, 99-111.

VALLADAS, H.; CACHIER, H.; MAURICE, P.; BERNALDO DE QUIRÓS, F.; CLOTTES, J.; CABRERA, V.; UZQUIANO, P. y ARNOLD, M.

1992 Direct radiocarbon dates for prehistoric paintings at the Altamira, El Castillo and Niaux caves. Nature 357, 68-70.

VILLAVERDE, V.

1994 Arte paleolíticos de la Cova del Parpalló. Estudio de la colección de plaquetas y cantos con grabados y pinturas. Tomo I y II. Servicio de investigación prehistórica. Diputación de Valencia. Valencia.

ZERVOS, C

1959 L'Art de l'Epoque du Renne en France. Éditions Cahiers d'Art. Paris.

ZILHÃO, J.

2003 Vers une chronologie plus fine du cycle ancien de l'art paléolithique de la Côa: quelques hypothèses de travail. In: Balbín, R. y Bueno, P. (eds): El arte prehistórico desde los inicios del siglo XXI. Primer Symposium Internacional de Arte Prehistórico de Ribadesella. Asociación Cultural de Amigos de Ribadesella. Ribadesella. 


\section{ANEXO I: Grafías analizadas}

\begin{tabular}{|c|c|c|}
\hline SIGLA & YACIMIENTO & REFERENCIA BIBLIOGRÁFICA \\
\hline TB1 & Tito Bustillo & CORCHÓN, 1986: 376, fig. 126.1 \\
\hline CA1 & El Castillo & CORCHÓN, 1986: 317, fig. 73 \\
\hline C1 & Covalanas & GUTIÉRREZ CUENCA et al., 2004: 11, fig. 2 \\
\hline L1 & Lortet & BARANDIARÁN, 2003: 150, fig. 28. \\
\hline CN1 & Candamo & HERNÁNDEZ PACHECO, 1919: 64, fig. 14 \\
\hline PP1 & Pair-non-Pair (1) & DELLUC y DELLUC, 2006: 23-46 (calco p. 34, fig. 48). \\
\hline PP2 & Pair-non-Pair (2) & DELLUC y DELLUC, 2006: 23-46 (calco p. 34, fig. 48). \\
\hline RS1 & Roc-aux-Sociers (1) & IAKOVLEVA y PINÇON, 1997: 60, fig. 69 \\
\hline RS2 & Roc-aux-Sociers (2) & IAKOVLEVA y PINÇON, 1997: 40, fig. 23 \\
\hline PE1 & Penascosa (1) & BAPTISTA, 1999: 99 \\
\hline PE2 & Penascosa (2) & BAPTISTA, 1999: 99 (detalle del caballo) \\
\hline E1 & Quinta da Barça (1) & BAPTISTA, 1999: 12. \\
\hline AX1 & Altxerri A & ALTUNA y APELLÁNIZ, 1976: 22, fig. 10 \\
\hline GE1 & Genovesi (1) & PALMA DI CESNOLA, 1993: 491, fig. 88.1 \\
\hline GE2 & Genovesi (2) & PALMA DI CESNOLA, 1993: 491, fig. 88.4 \\
\hline MA1 & Mas d'Azil (1) & CLOTTES, 2001: 56: 53-62. (Calco p. 53, fig. 1) \\
\hline BD1 & Bédeilhac & BANDI, 1988: 133-147 (Dibujo de Bürki, en p. 137, fig. 2) \\
\hline LM1 & La Madelaine & LEROI-GOURHAN, 1971: fig. 201 \\
\hline LA1 & Labastide & FRITZ et al., 2007: 164-181 (p. 175, fig. 4.9) \\
\hline MA2 & Mas d'Azil (2) & FRITZ et al., 2007: 164-181 (p. 175, fig. 4.10) \\
\hline MA3 & Mas d'Azil (3) & DELPORTE, 1990: 78, fig. 44 \\
\hline LB1 & Laugerie-Basse (1) & SIEVEKING, 1987b: 3, plate. 1.a \\
\hline LB2 & Laugerie-Basse (2) & MÜLLER-KARPE, 1982: 79. 17a \\
\hline LB3 & Laugerie-Basse (3) & SIEVEKING, 1987a: plate 10 y 11 \\
\hline Lu1 & Limeuil & TOSELLO, 2003: 125, fig. 71 \\
\hline TF1 & Trois-Frères (1) & BĖGOUËN y BREUIL, 1958: 59, fig. 63 \\
\hline TF2 & Trois-Frères (2) & BĖGOUËN y BREUIL, 1958: 51, fig. 55 \\
\hline AL1 & Altamira & BREUIL y OBERMAIER, 1984: 50-51 \\
\hline PA1 & La Pasiega & GARATE, 2010: 169, fig. 101 \\
\hline GB1 & Gabillou & GAUSSEN, 1964: planche 34.1 \\
\hline PS1 & Ribeira de Piscos (1) & BAPTISTA, 1999: 129 \\
\hline M1 & Montastruc & SIEVEKING, 1987a: plate 97 \\
\hline $\mathrm{F} 1$ & Figuier & SANCHIDRÍAN, 2001: 298, fig. 118.2 \\
\hline Mu1 & Murat (1) & LEMOZI, 1924: 22, fig. 3 \\
\hline Mu2 & Murat (2) & LEMOZI, 1924: 30, fig. 7 \\
\hline G1 & La Garma & GONZÁLEZ SAINZ, 2003: 216 \\
\hline LS1 & Laussel & DUHARD, 1996: 39, fig. 21 \\
\hline BQ1 & Bruniquel & SIEVEKING, 1987a: 94, fig 662 \\
\hline P1 & Parpalló & VILLAVERDE, 1994: fig. 144 (1778A) \\
\hline TF3 & Trois-Frères (3) & ZERVOS, 1959: 303, fig. 293 \\
\hline TB2 & Tito Bustillo (2) & BERENGUER, 1994: 135, fig. 107 \\
\hline SV1 & Siega Verde & ALCOLEA y BALBÍN, 2006: 93, fig. 51 \\
\hline CS1 & Los Casares & CABRÉ, 1940-1941: 87, fig. 4 \\
\hline TB3 & Tito Bustillo (3) & BALBíN et al., 2003: 197-108 \\
\hline GA2 & La Garma (2) & GONZÁLEZ SAINZ, 2003: 207, foto 1 \\
\hline Sa1 & Saalfeld & BOSINSKI, 1982: tafel 95.d \\
\hline Mh1 & La Marche (1) & AIRVAUX, 2001: 93, fig. 65 \\
\hline Mh2 & La Marche (2) & AIRVAUX, 2001: 93, fig. 65 \\
\hline LP1 & Le Placard & CLOTTES et al., 2010: 355, fig. 8 \\
\hline R1 & Doña Trinidad (1) & CANTALEJO et al., 2006: fig. IV.A.3 \\
\hline $\mathrm{R} 2$ & Doña Trinidad (2) & CANTALEJO et al., 2006: fig. IV.A.5 \\
\hline GG1 & La Griega & CORCHÓN, 1997: 60 \\
\hline Fs1 & Fariseu & BAPTISTA, 2009: 47 \\
\hline E2 & Quinta da Barca (2) & BAPTISTA, 2009: 150 \\
\hline PS2 & Ribeira de Piscos (2) & BAPTISTA, 1999: 129; ZILHÃO, 2003: 80 \\
\hline
\end{tabular}


